REVIEW

\title{
Endocannabinoids and exercise
}

\author{
A Dietrich, W F McDaniel
}

Br J Sports Med 2004;38:536-541. doi: 10.1136/bjsm.2004.011718

Exercise induces changes in mental status, particularly analgesia, sedation, anxiolysis, and a sense of wellbeing. The mechanisms underlying these changes remain unknown. Recent findings show that exercise increases serum concentrations of endocannabinoids, suggesting a possible explanation for a number of these changes. This article provides an overview of this emerging field.

See end of article for authors' affiliations

Correspondence to Dr Dietrich, PO Box 110236, Riad El-Solh, Beirut 1107-2020, Lebanon; arnedietrich@aub.edu.lb

Correspondence to: Dr McDaniel, Georgia College and State University, Milledgeville, GA 31061, USA; bill.mcdaniel@gcsu.edu

Accepted 4 May 2004
A $n$ exercise induced altered state of consciousness has long been appreciated by endurance athletes. The effect has been well documented in the popular literature and subjected to scientific investigation. ${ }^{1-3}$ In the late 1960s, the psychological changes associated with prolonged physical activity were often described as a "second wind."4 A more contemporary label often applied to these exercise induced changes is the "runner's high." The runner's high has been described subjectively as pure happiness, elation, a feeling of unity with one's self and/or nature, endless peacefulness, inner harmony, boundless energy, and a reduction in pain sensation. $^{5-9}$ These subjective descriptions are similar to the claims of distorted perception, atypical thought patterns, diminished awareness of one's surroundings, and intensified introspective understanding of one's sense of identity and emotional status made by people who describe drug or trance states.

As is the case with all phenomena related to consciousness and its alterations, the runner's high is a private experience, and the evidence for its existence rests predominantly on verbal report. Scientific inquiry into the phenomenon has been restricted even further because of its ephemeral nature. For example, the runner's high is not experienced by all runners, and this experience does not occur consistently in runners who have experienced it previously. These observations have left laymen and scientists wondering why and under which conditions the runner's high occurs, or whether or not it exists at all.

Before the discovery of the opioids, exercise scientists tried to account for the analgesic and euphoric mental states with alterations in the catecholamines adrenaline (epinephrine) and noradrenaline (norepinephrine). ${ }^{10}$ With the discovery and subsequent characterization of the opioid receptor network and endogenous opioid peptides, an entirely different mechanism of action evolved. Soon thereafter, exercise induced changes in psychological functions were often described as being a direct consequence of alterations in endogenous opioid release.
However, there are a number of serious problems with the "endorphin hypothesis."11 12 Studies examining the exercise-endorphin connection produced equivocal results, and many of the studies were plagued by methodological confounds. For instance, $\beta$ endorphin has almost the same amino acid sequence as other members of the pro-opiomelanocortin family such as the adrenocorticotrophic hormone, making cross reactivity to the detecting antibody a serious confound. Also, adrenocorticotrophic hormone is a stress hormone that is known to increase with exercise, compounding the problem. There are also major inconsistencies between the endorphin hypothesis and the physiological and biochemical responses to endurance exercise. For instance, $\beta$ endorphins bind best to the $\mu$ opioid receptor, the endogenous opioid system that mediates the analgesic and euphoric properties of the opiates. However, minimal activation of the same endogenous opioid system is also responsible for the severe respiratory depression, pinpoint pupils, and inhibition of gastrointestinal motility, all of which accompany opiate use. Yet, these effects are not seen in runners. The most limiting factor, however, is that the endorphin hypothesis rests entirely on research measuring endorphins in circulating blood, as ethical reasons preclude the determination of central concentrations of endorphins. Because endorphins are too large to cross the blood-brain barrier, peripheral activation in the systemic circulation cannot be taken as indicative of central effects. In recent years, several prominent endorphin researchers-for example, Dr Huda Akil and Dr Solomon Snyder-have publicly criticised the hypothesis as being "overly simplistic", being "poorly supported by scientific evidence", and a "myth perpetrated by pop culture."11

At first glance, it appears that the runner's high phenomenon is, at present, not a scientific problem because it is built on circumstantial evidence and lacks a plausible mechanistic explanation. However, recent data in our laboratory showed that endurance exercise activates the endocannabinoid system, ${ }^{13}$ suggesting a new mechanism underlying exercise induced alterations of mental status. Using trained male college students running on a treadmill or cycling on a stationary bike for 50 minutes at $70-80 \%$ of maximum heart rate, we found that exercise of moderate intensity dramatically increased concentrations of anandamide in blood plasma. (Currently, research is underway in our laboratory to explore this finding further by examining

Abbreviations: 2-AG, sn-2-arachidonylglycerol; THC, tetrahydrocannabinol 
the effect of exercise on both serum and cerebrospinal fluid concentrations of endocannabinoids in exercising rats, while also examining several associated alterations of behaviour.) Because activation of the endocannabinoid system reduces pain sensations ${ }^{14}$ and alters emotional and cognitive processes, $^{15}{ }^{16}$ this finding has implication for some of the psychological effects that accompany exercise. Owing to the presence of cannabinoid receptors in muscle, skin, endothelial cells, and lung, ${ }^{17-19}$ this finding also suggests a possible role for the endocannabinoid system in mediating certain physiological responses to exercise.

It is important to emphasise that the intention of this paper is not to substitute one neurotransmitter for another and perpetuate the simple reductionist idea of one neurochemical being responsible for a complex variety of psychological processes. Rather, we review the literature on the functional role of the endocannabinoid system as it relates to exercise and call attention to the possibility that the endocannabinoid system may play an important role in the physiological and psychological adaptations to exercise. The review opens unexpected and entirely novel avenues of research in exercise physiology and psychology and is offered on the strength of its heuristic value.

In addition, we propose to reconceptualise the runner's high into a set of behavioural phenomena that can be, at least to a large extent, subjected to scientific scrutiny. Traditionally, the runner's high has been operationally defined as a "euphoric sensation experienced during running, usually unexpected, in which the runner feels a heightened sense of well being, enhanced appreciation of nature, and transcendence of barriers of time and space"

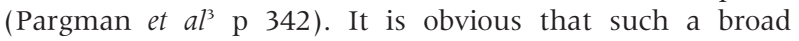
definition, in conjunction with the extensive use of esoteric language, does not qualify as an operational definition that can be used to derive testable hypotheses. We propose instead a more limited operational definition of the runner's high centred mostly on observable behaviours such as analgesia, sedation (post-exercise calm), anxiolysis, and a sense of wellbeing. This definition has a number of advantages. Firstly, there is a large body of scientific literature documenting that exercise suppresses pain, ${ }^{20}$ induces sedation, ${ }^{21}$ reduces stress, ${ }^{22}$ and elevates mood. ${ }^{23}{ }^{24}$ Secondly, because these effects are directly measurable, the operational definition allows the formulation of empirical predictions and the testing of specific hypotheses. Moreover, data from animal research can be recruited to elucidate the phenomena, as exercise in rodents has been shown to increase tolerance of pain (hot plate or tail flick tests), induce sedation (open field test), and produce anxiolysis (elevated plus maze).

\section{THE ENDOCANNABINOID HYPOTHESIS}

Endocannabinoid receptors and their endogenous ligands have been identified. ${ }^{25-28}$ To date, two cannabinoid receptor subtypes have been cloned. The $\mathrm{CB}_{1}$ receptor is located in the central nervous system, and it is more densely concentrated on the membranes of neurones located in the cortex, hippocampus, basal ganglia, amygdala, hypothalamus, and cerebellum. $^{29} \mathrm{CB}_{1}$ receptors are also found in several peripheral sites, including the peripheral nervous system. ${ }^{31}$ The $\mathrm{CB}_{2}$ receptor, on the other hand, is located mainly in peripheral tissue. Both the $\mathrm{CB}_{1}$ and $\mathrm{CB}_{2}$ receptors are coupled to $\mathrm{Gi} / \mathrm{o}$ proteins. Thus cannabinoid receptors inhibit adenylate cyclase and, thereby, depending on the cell type, either inhibit voltage gated calcium channels or activate potassium channels. ${ }^{32}$ Thus, with respect to the nervous system, the general effect of $\mathrm{CB}_{1}$ activation is neuronal inhibition, which does not apply to $\mathrm{CB}_{2}$ receptors, as they are mainly expressed on immune cells. There is also an ongoing hypothesis in the field that there may exist an additional cannabinoid receptor, tentatively named $\mathrm{CB}_{3} \cdot{ }^{33}$ Although the existence of a $\mathrm{CB}_{3}$ receptor is currently hypothetical, it may be of interest as some of the effects reported in this review might turn out not to be accounted for by $\mathrm{CB}_{1}$ and $\mathrm{CB}_{2}$ receptors.

Two naturally occurring ligands, which are members of a small family of fatty acid derivatives, have been identified for $\mathrm{CB}_{1}$ and $\mathrm{CB}_{2}$ receptors. Anandamide is one ligand, and it exhibits a higher affinity for the $\mathrm{CB}_{1}$ receptor subtype than $\mathrm{CB}_{2} \cdot{ }^{25}$ A second ligand, sn-2-arachidonylglycerol (2-AG), has been identified more recently. ${ }^{27}$ Although the two endocannabinoids are found in the systemic circulation at equal concentrations, the concentration of 2-AG is about 200 times higher than that of anandamide in the brain. ${ }^{34}$ Anandamide and 2-AG have different biosynthetic pathways and may be produced under different conditions. ${ }^{34}$ However, the sites of anandamide and 2-AG production in brain and peripheral tissues are not known. Because endocannabinoids are lipids that are rapidly eliminated from extracellular space, it is generally assumed that production sites are located in close proximity to their attending cannabinergic receptors. More importantly, the environmental stimuli responsible for the production and release of endocannabinoids are also unknown, making it difficult to assess the physiological and behavioural functions of anandamide and 2-AG.

\section{Cannabinoids and exercise induced analgesia}

The role of the endocannabinoid system as an alternative neuromodulatory system in pain perception has been a central focus of cannabinoid research. ${ }^{14} 3035$ Analgesia is mediated in part by the endogenous opioid system. However, analgesia that is insensitive to opioid antagonists can also occur, providing evidence for non-opioid antinociception. Using animal models of acute and tonic pain, behavioural studies with a wide variety of noxious stimuli have shown cannabinoid induced antinociception, ${ }^{36}$ which is mediated by $\mathrm{CB}_{1}$ receptors. ${ }^{30}$ The potency and efficacy of cannabinoids in producing antinociception rivals that of morphine.

Unlike opioid mediated suppression of pain neurotransmission, the endocannabinoid system has been shown to suppress pain not only at central, but also at peripheral concentrations. ${ }^{14}{ }^{19} \mathrm{CB}_{1}$ receptors are densely expressed on peripheral nerve terminals such as pain sensing $C$ (small diameter) fibres, large diameter $A ß$ and $A \delta$ fibres, as well as in the dorsal root ganglia. ${ }^{37} 38$

Cannabinoids also act at central sites to modulate pain signalling. For example, cannabinoid receptors in the dorsal horn of the lumbar spinal cord have been shown to attenuate pain evoked by noxious heat applied to rat hind paw. ${ }^{36}$ In the brain, Meng et $a^{35}$ found a brainstem circuit involving the rostral ventromedial medulla that is activated by cannabinoids. Although activation of neurones in the rostral ventromedial medulla is also required for the analgesic effects of morphine, the cannabinoids modulate its activity independently, demonstrating a separate central mechanism of action for antinociception. The cannabinoids also affect pain perception by acting in the periaqueductal gray system, an area dense in opioid receptors. ${ }^{39}$ Electrical stimulation of the dorsal and lateral periaqueductal gray system produces analgesia that is both $\mathrm{CB}_{1}$ receptor mediated and accompanied by the release of anandamide in the system. Finally, subcutaneous injection of the chemical irritant formalin triggers an increase of anandamide in the periaqueductal gray system, further implicating the endocannabinoids not only in the modulation of chemogenic pain, but also more generally in the centrally mediated suppression of pain. ${ }^{39}$ Cannabinoids and opioids also exhibit synergistic effects in the production of antinociception, and bidirectional interactions between opioid and cannabinoid dependence have been reported. For example, administration of naloxone, a $\mu$ opioid 
receptor antagonist, induces withdrawal in cannabinoid dependent rats, whereas administration of the $\mathrm{CB}_{1}$ receptor antagonist SR141716A precipitates withdrawal in morphine dependent rats. ${ }^{40}$

With regard to exercise induced analgesia, there are some significant differences between opioid and cannabinoid antinociception. Firstly, cannabinoids produce analgesia by acting at a number of peripheral sites. ${ }^{14}{ }^{19}$ Although endocannabinoids such as anandamide are lipids and can cross the blood-brain barrier readily, this is not a requirement for the analgesic properties of endocannabinoids. This fact avoids one of the principal problems that plagued the endorphin hypothesis of exercise induced analgesia. Secondly, because of its highly lipophilic properties, systemic increases in anandamide concentrations are generally assumed to produce central effects. Consequently, in addition to peripheral sites, the increase in blood anandamide concentrations in endurance athletes is likely to activate analgesic systems in the brain. Finally, as mentioned above, subcutaneous injections of the chemical irritant formalin into rat hind paw increases the release of anandamide in the periaqueductal gray system, ${ }^{39}$ showing that noxious agents can produce analgesia at central sites without the activation of peripherally circulating endocannabinoids.

Research on cannabinoid induced analgesia has made use of a variety of noxious stimuli, and it has become clear that different types of tissue damage (mechanical, thermal, chemical, etc) differentially activate the endocannabinoid system. ${ }^{14}{ }^{19}$ The finding that there are particular types of pain against which cannabinoids are particularly effective may provide fresh insights into the sport specificity of the runner's high. It is curious that an "exercise high", similar to the one experienced by long distance runners, should not occur in athletic activities involving brief physical exertion, such as sprinting and weightlifting, or in sports requiring changes in pace and workload such as track, soccer, football, tennis, basketball, etc. Further testing should resolve the issue whether these activities engage the endocannabinoid system. Yet, there is also no reference to a "swimmer's high" in the literature, although it is a rhythmic and repetitive activity producing a particular pain concentration at a specific heart rate. Bearing on this problem, evidence is accumulating that cannabinoids induce analgesia by acting through $\mathrm{CB}_{1}$ receptors located in skin. ${ }^{19}{ }^{19}$ This mechanism might suggest that painful stimuli to the skin are particularly potent in activating endocannabinoid antinociception. Unlike other rhythmic endurance activities such as swimming, running is a weight bearing sport in which the feet must absorb the "pounding of the pavement." We are not arguing that moderate intensity long distance swimming fails to activate the endocannabinoid system. Rather, an endurance activity of this nature may not stimulate endocannabinoid release to as great an extent as running.

It is also important to mention with regard to the runner's high that cannabinoids produce neither the respiratory depression, meiosis, or strong inhibition of gastrointestinal motility associated with opiates and opioids. This is because there are few $\mathrm{CB}_{1}$ receptors in the brainstem ${ }^{42}$ and, apparently, the large intestine.

Finally, anandamide also inhibits oedema and inflammation, ${ }^{19}$ and low doses of cannabinoids of insufficient magnitude to produce analgesia or motor impairment ${ }^{43}$ attenuate chemogenic pain. ${ }^{44}$ This observation is also relevant to exercise induced analgesia, as muscle pain is believed, in part, to be the result of the generation of substances such as lactic acid. ${ }^{20}$

\section{Psychoactive effects of cannabinoids}

The psychoactive constituent of marijuana, $\Delta$-(9)-tetrahydrocannabinol (THC), exhibits high affinity for the $\mathrm{CB}_{1}$ receptor, which is densely expressed in brain regions implicated in the control of emotion and cognition..$^{29}$ This distribution provides the basis for the profound psychological effects of exogenous cannabinoids. A prominent effect of cannabinoids is the induction of sedation. ${ }^{15}$ In addition, cannabinoids are reported to reduce anxiety, ${ }^{16}$ alter attention, ${ }^{45}$ and impair both working memory ${ }^{46}$ and spatial learning, ${ }^{47} 48$ apparently by interfering with hippocampus dependent neuronal processes responsible for declarative memory. ${ }^{49}$ Users of marijuana often report distortions of time estimation,, ${ }^{50}$ euphoria and enhanced sensory perception,,${ }^{51}$ a state of silent introspection, and feelings of wellbeing. ${ }^{16}{ }^{45}$

Cannabinoids exert a negative effect on dopaminergic activity in the prefrontal cortex. ${ }^{52}$ For example, treatment with THC results in a change in regional cerebral blood flow in the rat. In particular, decreases have been measured to the hippocampus and the frontal and medial prefrontal cortices. However, changes have not been found in the ventral tegmentum, caudate nucleus, cerebellum, temporal cortex, parietal cortex, or occipital cortex..$^{53}$ Likewise, as evidenced by functional nuclear magnetic resonance imaging, chronic marijuana users show decreased activity of the dorsolateral prefrontal cortex, an area highly associated with working memory. ${ }^{54}$ A decrease in prefrontal cortex metabolism has also been shown in rats chronically exposed to THC. ${ }^{55}$ It has been suggested ${ }^{52}$ that hypometabolism in prefrontal cortical regions may contribute significantly to the impaired cognitive processes associated with cannabinoid use.

Administration of the endogenous cannabinoid anandamide, which also binds to $\mathrm{CB}_{1}$ receptor, elicits similar effects to those produced by THC. ${ }^{56}$ Although some pharmacological differences exist between the plant derived THC and anandamide, systematic structure-activity relation studies have shown that the two compounds act at the $\mathrm{CB}_{1}$ receptor in a similar manner. ${ }^{56}$

The intense psychological experiences elicited by the activation of the endocannabinoid receptors are strikingly similar to the experience of the runner's high. To compare, the mental changes that accompany long distance running include analgesia, sedation (post-exercise calm or glow), a reduction in anxiety, euphoria, and difficulties in estimating the passage of time. ${ }^{32021}$ In addition, a recent study investigating higher cognitive functioning during exercise has shown that prolonged running and cycling produces deficiencies in prefrontal dependent cognitive processes such as sustained attention and working memory. ${ }^{57}$ One possible explanation of these findings may be that the increased endocannabinoid release during exercise results in diminished metabolism in prefrontal regions while at the same time altering cognitive function and consciousness. Although such parallels are anecdotal and speculative, such comparisons have shed light on psychological and pharmacological phenomena in the past. As with the mental changes associated with long distance running, most of the behavioural effects of the cannabinoids depend on set and setting.

\section{Cannabinoids and motor behaviour}

The highest concentration of $\mathrm{CB}_{1}$ receptors in the brain can be found in the basal ganglia, particularly in output nuclei, and the cerebellum, implicating the endocannabinoid system in the control of movement. ${ }^{29}{ }^{42}$ Indeed, there is substantial evidence that cannabinoids affect motor behaviour. ${ }^{42} 4358$

In a variety of species including humans, administration of plant derived endogenous and synthetic cannabinoids produces biphasic effects on locomotion. ${ }^{15}{ }^{43}$ In larger doses, cannabinoid agonists produce well known and profound motor inhibition. Thus, as might be expected, cannabinoids have proven clinically useful in treating movement disorders such as tics, dyskinesia, tremors, and dystonia. ${ }^{1659}$ These 
effects are reversed by administration of the selective $\mathrm{CB}_{1}$ receptor antagonist SR141716A, providing evidence that cannabinoids mediate motor activity through the $\mathrm{CB}_{1}$ receptor. In low doses, however, cannabinoids tend to produce hyperactivity. ${ }^{18}{ }^{43}{ }^{60}$ For example, intracerebroventricular injection of low doses of anandamide increased locomotion, and systemic injections of low doses of anandamide stimulated behavioural activity in the open field ${ }^{43} 58$ Finally, $\mathrm{CB}_{1}$ knockout mice are extremely hypoactive, ${ }^{61}$ suggesting that one of the principal physiological roles of the endocannabinoid system may be the refinement of movements needed for coordinated locomotion.

Cannabinoids appear to modulate motor behaviour by altering the transmission of other transmitter systems in the basal ganglia. They appear to increase $\gamma$ aminobutyric acid transmission (although this remains controversial), inhibit glutamate transmission, and inhibit the reuptake of dopamine. $^{3362}$ In addition, anandamide release in the dorsal striatum increases in freely moving rats, and it has been suggested that it acts as a striatal neurotransmitter in its own right $^{63}$ (for a thorough review, see Piomelli3 ${ }^{33}$.

It is worth noting that the basal ganglia have been implicated in the control of movements based on well learned motor behaviour. ${ }^{64}$ The more a motor skill is practiced and becomes automatic, the more the details of its execution come under the control of the basal ganglia. ${ }^{65}$ Hence, we predict that low level skills such as running, which are controlled to a higher degree by the basal ganglia than high level skills, such as basketball, hockey, or tennis, may more readily activate the endocannabinoid system.

\section{Cannabinoids and addiction}

There is also a close interaction between dopamine and the endocannabinoid system in structures that are implicated in the brain's reward system. The dopamine $\mathrm{D}_{1}$ and $\mathrm{D}_{2}$ receptors are colocalised with the $\mathrm{CB}_{1}$ receptor, ${ }^{66}$ and it has been shown that the cannabinoids alter dopaminergic activity in the medial forebrain bundle. ${ }^{54}$ Cannabinoids increase the firing rates of dopaminergic neurones in the ventral tegmentum, substantia nigra, and the medial forebrain bundle. ${ }^{67-69}$ Withdrawal from cannabinoids, on the other hand, results in a decline in dopaminergic activity in the medial forebrain bundle. $^{70}$ Projections from the ventral tegmentum to the nucleus accumbens via the median forebrain bundle mediate the rewarding effects of most drugs of abuse, and the reinforcing properties of endocannabinoids and cannabinoids appear to be mediated through this pathway. ${ }^{68}$ Evidence now supports the hypothesis that both endocannabinoids and exogenous cannabinoids induce a selective release of dopamine in the shell of nucleus accumbens through $\mathrm{CB}_{1}$ receptors. ${ }^{32}$ This evidence also suggests that the activation of endogenous cannabinoids through exercise could account for exercise addiction. It also follows that pretreatment with selective dopamine or endocannabinoid receptor antagonists should block induction of an exercise induced high. These possibilities deserve scientific inquiry, although the ethics of such studies in humans would bring into question morale issues.

Long distance running has been described as "addictive."71 This is not unlike the anecdotal "withdrawal" experiences usually described as a dysphoric state by many runners when forced by knee problems to switch to an alternative activity. Recent studies have resolved a long debated argument about the addictive properties of cannabinoids (for a thorough review, see Maldonado and Rodríguez de Fonseca ${ }^{72}$ ). Rodents previously exposed chronically to high doses of THC and then treated with a $\mathrm{CB}_{1}$ receptor antagonist such as SR141716A do, in fact, show signs of withdrawal syndrome. ${ }^{72}$ The somatic signs of withdrawal include wet dog shakes, front paw tremor, body tremor, hypolocomotion, ataxia, mastication, piloerection, excessive licking, scratching, and rubbing. It has been suggested that the failure to observe severe withdrawal symptoms in humans chronically exposed to marijuana may relate to the long half life of THC and its metabolites. This is compounded by the fact that THC and its psychoactive metabolites are extremely lipophilic. Thus, they are stored in fatty tissues and released slowly into the blood stream once abstinence is experienced. Collectively, these factors could counter acute withdrawal symptoms when chronic use of cannabis is terminated in both non-humans and humans. ${ }^{32} 73$

Finally, increased corticotrophin releasing factor, and inhibition of mesolimbic dopamine activity is a common withdrawal symptom produced by many drugs of abuse. ${ }^{74}$ Animals undergoing SR141716A induced cannabinoid withdrawal show increased corticotrophin releasing factor release and enhanced Fos activity in the central amygdala. ${ }^{67}$ It has been hypothesised ${ }^{58}$ that this change in limbic system corticotrophin releasing factor function could be responsible for the increased feelings of anxiety and depression that often accompanies cannabinoid withdrawal. This, along with the reduction in spontaneous firing of DA neurones in the ventral tegmentum during cannabinoid abstinence, ${ }^{70}$ is thought to be the likely physiological mechanism responsible for the dysphoria associated with cannabis withdrawal. ${ }^{72}$ We do not suggest that the addictive aspects of exercise are identical with the addictive properties of exogenous cannabinoids, but that a similarity exists between the desire that some people have for exercise and the desire they may have for exogenous administrations of cannabinoids. The parallels between these results and the subjective experiences associated with exercise abstinence, as discussed previously, are considerable.

\section{Peripheral effects}

Activation of the endocannabinoid system may also participate in other adaptive responses to exercise. For instance, anandamide acts as a vasodilator and produces hypoten$\operatorname{sion}^{76-78}$ and may thus facilitate blood flow during exercise. Although the distribution of $\mathrm{CB}_{1}$ receptors in smooth muscle and endothelial cells suggests that the vasorelaxant effects of anandamide are mediated through $\mathrm{CB}_{1}$ or $\mathrm{CB}_{2}$ receptors, recent experiments ${ }^{79}$ have intimated a prominent role of vanilloid receptors in the vasodilatory effects of these endocannabinoids. Finally, cannabinoids affect the respiratory system. Although studies have reported bidirectional control of airway responsiveness, in general, endocannabinoids and exogenous cannabinoids act as bronchodilators. ${ }^{17}$ Consequently, a possible role for the endocannabinoid system could be to facilitate breathing during exercise.

\section{CONCLUSIONS}

To date, a sound neural mechanism for the well known beneficial effects of exercise on mental health has yet to be proposed. Recent findings show that exercise increases serum concentrations of endocannabinoids, a result suggestive of a new possible explanation for a number of these changes. Further research is necessary to characterise the precise nature of this endocannabinoid response to exercise, specifically the relative importance of factors such as the nature of the activity, exercise duration, exercise intensity, sex, and age. In addition, animal models can be used to identify the production and binding sites of endocannabinoids as well as their functional role in exercise.

The cannabinoids produce psychological states that closely parallel several experiences described as being related to the runner's high. Compared with the opioid analgesics, the analgesia produced by the endocannabinoid system is more consistent with exercise induced analgesia. Activation of the 
endocannabinoid system also produces sedation, anxiolysis, a sense of wellbeing, reduced attentional capacity, impaired working memory ability, and difficulty in time estimation. This behavioural profile is similar to the psychological experiences reported by long distance runners. Considerable research is needed to clarify to what extent the endocannabinoid system might be responsible for the exercise induced changes in mental status. Nevertheless, a significant upregulation of serum concentrations of endocannabinoids has recently been reported in endurance athletes, and studies are underway to explore this further in laboratory animals.

The close interaction of endocannabinoids with dopamine shows that they have a function in the brain's reward system and therefore possibly addiction. The endocannabinoid system is also implicated in the control of motor activity mediated through the basal ganglia, and central activation of anandamide in freely moving rats has been demonstrated. ${ }^{63}$ Finally, the endocannabinoid system mediates peripheral effects such as vasodilation and bronchodilation that may play a contributory role in the body's response to exercise.

This article is intended to provide an overview of the emerging field of the endocannabinoid-exercise interaction. The list of topics was necessarily selective, but it is offered in the hope that researchers of diverse backgrounds will use the review to conduct empirical tests of its premises. We suggest that the "endocannabinoid hypothesis" is a feasible alternative to the endorphin hypothesis and should be subjected to further empirical tests.

\section{Authors' affiliations}

A Dietrich, Department of Social and Behavioral Sciences, American University of Beirut, Lebanon

W F McDaniel, Georgia College and State University, Milledgeville, GA, USA

\section{REFERENCES}

1 Carmack MA, Martens R. Measuring commitment to running: a survey of runners' attitudes and mental states. J Sports Psychol 1979;1:25-42.

2 Dietrich A. Functional neuroanatomy of altered states of consciousness: the transient hypofrontality hypothesis. Conscious Cogn 2003;12:231-56.

3 Pargman D, Baker M. Running high: enkephalin addicted. J Drug Issues 1980;10:341-9.

4 Mandell AJ. The second wind. In: Sacks MH, Sachs ML, eds. Psychology of running. Champaign, IL: Human Kinetics, 1981:221-3.

5 Farrell PA, Gustafson AB, Morgan WP, et al. Enkephalins, catecholamines, and psychological mood alterations: effects of prolonged exercise. Med Sci Sports Exerc 1987; 19:347-53.

6 Glasser W. Positive addiction. New York: Harper and Row, 1976.

7 Hoffman P. The endorphin hypothesis. In: Morgan WP, eds. Physical activity \& mental health. Washington: Taylor \& Francis, 1997:161-77.

8 Kostrubala T. The joy of running. Philadelphia: JB Lippincott, 1976:51.

9 Sheehan GA. Running and being: a total experience. New York: Simon and Schuster, 1978

10 Howley ET. The effect of different intensities on exercise on the excretion of epinephrine and norepinephrine. Med Sci Sports Exerc 1976;8:219-22.

11 Kolata G. Runners high? Endorphins? Fiction say some scientists. The NY Times, 2002 May, 21

12 Schlicht W. Sport und Primärprävention. Göttingen: Hogrefe, 1994.

13 Sparling PB, Giuffrida A, Piomelli D, et al. Exercise activates the endocannabinoid system. Neuroreport, 2003;14, 2209-11.

14 Richardson JD. Cannabinoids modulate pain by multiple mechanisms of action. J Pain 2000;1:2-14.

15 Chaperon F, Thiebot MH. Behavioral effects of cannabinoid agents in animals. Crit Rev Neurobiol 1999;13:243-81.

16 Diaz J. How drugs influence behavior: a neurobehavioral approach. Upper Saddle River, NJ: Prentice Hall, 1997.

17 Calignano A, Katona I, Desarnaud MH, et al. Bidirectional control of airway responsiveness by endogenous cannabinoids. Nature 2000;408:96-101.

18 Di Marzo V, De Petrocellis L, Fezza, F, et al. Anandamide receptors. Prostaglandins Leukot Essent Fatty Acids 2002;66:377-91

19 Richardson JD, Kilo S, Hargreaves KM. Cannabinoids reduce hyperalgesia and inflammation via interaction with peripheral $C_{1}$ receptors. Pain 1998;75:111-19.

20 Cook DB, Koltyn KF. Pain and exercise. Int J Sport Psychol 2000;31:256-77

21 Salmon P. Effects of physical exercise on anxiety, depression, and sensitivity to stress: a unifying theory. Clin Psychol Rev 2001;21:33-61

22 Paluska SA, Schwenk TL. Physical activity and mental health: current concepts. Sports Med 2000;29: 167-80.
23 Craft LL, Landers DM. The effect of exercise on clinical depression resulting from mental illness: a meta-analysis. Journal of Sports \& Exercise Psychology 1994;20:339-57.

24 Scully D, Kremer J, Meade M. M, et al. Physical exercise and psychological well being: a critical review, $\mathrm{Br} J$ Sports Med 1998:32:111-20.

25 Devane WA, Hanus $L$, Breuer A, et al. Isolation and structure of a brain constituent that binds to the cannabinoid receptor. Science 1992;258:1946-9.

26 Matsuda LA, Lolait SJ, Brownstein MJ, et al. Structure of a cannabinoid receptor and functional expression of the cloned cDNA. Nature 1990;346:561-4.

27 Mechoulam R, Benshabat S, Hanus L, et al. Identification of an endogenous 2monoglyceride, present in canine gut, that binds to cannabinoid receptors. Biochem Pharmacol 1995:50:83-90.

28 Munro S, Thomas KL, Abu-Shaar M. Molecular characterization of a peripheral receptor for cannabinoids. Nature 1993;365:61-5

29 Glass M, Dragunow M, Faull RLM. Cannabinoid receptors in the human brain: a detailed anatomical and quantitative autoradiographic study in the fetal, neonatal and adult human brain. Neuroscience 1997;10:1665-9.

30 Pertwee RG. Cannabinoid receptors and pain. Prog Neurobiol 2001;63:569-611.

31 Lynn $A B$, Herkenham M. Localization of cannabinoid receptors and nonsaturable high-density cannabinoid binding sites in peripheral tissues of the rat: Implications for receptor-mediated immune modulation by cannabinoids. J Pharmacol Exp Ther 1994;268:1612-23.

32 Nestler EJ, Hyman SE, Malenka RC. Molecular neuropharmacology: a foundation for clinical neuroscience. New York: McGraw-Hill, 2001

33 Piomelli $\mathbf{D}$. The molecular logic of endocannabinoid signalling. Nat Rev Neurosci 2003;4:873-84.

34 Stella N, Schweitzer P, Piomelli D. A second endogenous cannabinoid that modulates long-term potentiation. Nature 1997;388:773-8.

35 Meng ID, Manning BH, Martin WJ, et al. An analgesia circuit activated by cannabinoids. Nature 1998:395:381-3.

36 Hohmann AG, Tsou K, Walker JM. Cannabinoid suppression of noxious heatevoked activity in wide dynamic range neurons in the lumbar dorsal horn of the rat. J Neurophysiol, 1999;81:575-83.

37 Hohmann AG, Herkenham M. Localization of central cannabinoid $\mathrm{CB}_{1}$ receptor messenger RNA in neuronal subpopulations of rat dorsal root ganglia: a double label in situ hybridization study. NeurosciENCE 1999;90:923-31.

38 Ong YW, Mackie K. A light electron microscope study of the $\mathrm{CB}_{1}$ cannabinoid receptor in primate brain. Neuroscience 1999:92:1177-91.

39 Walker JM, Huang SM, Strangman NM, et al. Pain modulation by release of the endogenous cannabinoid anandamide. Proc Natl Acad Sci USA 1999;96:12198-203

40 Navarro $M$, Chowen J, Carrera MRA, et al. $C_{1}$ cannabinoid receptor antagonist-induced opiate withdrawal in morphine-dependent rats. Neuroreport 1998;9:3397-402.

41 Calignano A, La Rana G, Giuffrida A, et al. Control of pain initiation by endogenous cannabinoids. Nature 1998;394:277-81.

42 Herkenham $M$, Lynn $A B$, Ross-Johnson $M$, et al. Characterization and localization of cannabinoid receptors in rat brain: a quantitive in vitro autoradiographic study. J Neurosci 1991;11:563-83.

43 Sulcova E, Mechoulam R, Fride E. Biphasic effects of anandamide. Pharmacol Biochem Behav 1998;59:347-52.

44 Li J, Daughters RS, Bullis $C$, et al. The cannabinoid receptor agonist WIN $55,212-2$ mesylate blocks the development of hyperalgesia produced by capsaicin in rats. Pain 1998;81:25-33

45 Dewey WL. Cannabinoid pharmacology. Pharmacol Rev. 1986;39: 151-78)

46 Braida D, Sala M. Cannabinoid-induced working memory impairment is reversed by a second generation cholinesterase inhibitor in rats. Neuroreport, 2000;1 1;2025-9

47 Fernández-Ruiz J, Berrendero F, Hernandez, ML, et al. The endogenous cannabinoid system and brain development. Trends Neurosci 2000;23:14-20.

48 Murillo-Rodríguez E, Sanchez-Alavez M, Navarro L, et al. Anandamide modulates sleep and memory in rats. Brain Res 1998;812:270-4.

49 Hampson RE, Deadwyler SA. Cannabinoids, hippocampal function and memory. Life Sci 1999:65:715-23.

50 Han CJ, Robinson JK. Cannabinoid modulation of time estimation in the rat Behav Neurosci 2001;115:243-6.

51 Ameri A. The effects of cannabinoids on the brain. Prog Neurobiol 1999;58:315-48.

52 Pistis M, Porcu G, Melis M, et al. Effects of cannabinoids on prefrontal neuronal responses to ventral tegmental area stimulation. Eur J Neurosci 2001;14:96-102.

53 Bloom AS, Tershner S, Fuller SA, et al. Cannabinoid-induced alterations in regional cerebral blood flow in the rat. Pharmacol Biochem Behav 1997; 57:625-31.

54 Loeber RT, Yurgelun-Todd DA. Human neuroimaging of acute and chronic marijuana use: implications for frontocerebellar dysfunction. Hum Psychopharmacol 1999; 14:291-304.

55 Jentsch JD, Verrico CD, Le D, et al. Repeated exposure to delta-9tetrahydrocannabinol reduces prefrontal cortical dopamine metabolism. Neurosci Lett 1998;246:169-72.

56 Martin BR, Mechoulam R, Razdan RK. Discovery and characterization of endogenous cannabinoids. Life Sci 1995;65:573-95.

57 Dietrich A, Sparling PB. Endurance exercise selectively impairs prefrontaldependent cognition. Brain Cogn 2004;55:516-24. 
58 Romero J, de Miguel R, García-Palomero E, et al. Time-course of the effects of anandamide, the putative endogenous cannabinoid ligand, on extrapyramidal function. Brain Res 1995;694:223-32.

59 Müller-Vahl KR, Kolbe H, Schneider U, et al. Cannabis in movement disorders. Forsch Komplementarmed 1999:6:23-7.

60 Señudo-Pena MC, Romero J, Seale GE, et al. Activational role of cannabinoids on movement. Eur J Pharmacol 2000;391:269-74.

61 Steiner H, Bonner TI, Zimmer AM, et al. Altered gene expression in striate projection neurons in $\mathrm{CB}_{1}$ cannabinoid receptor knockout mice. Proc Natl Acad Sci USA 1999:96:5786-90.

62 Fernández-Ruiz J, Lastres-Becker I, Cabranes A, et al. Endocannabinoids and basal ganglia functionality. Prostaglandins Leukot Essent Fatty Acids 2002;66:257-67.

63 Giuffrida A, Parsons LH, Kerr TM, et al. Dopamine activation of endogenous cannabinoid signaling in dorsal striatum. Nat Neurosci 1999;2:358-63.

64 Mishkin M, Malamut B, Bachevalier J. Memory and habit: two neural systems. In: Lynch G, McGaugh JJ, Weinberger NM, eds. Neurobiology of learning and memory. New York: Guilford Press, 1984:66-77.

65 Jenkins IH, Brooks DJ, Nixon PD, et al. Motor sequence learning: a study with positron emission tomography. J Neurosci 1994;14:3775-90.

66 Hohmann AG, Herkenham M. Localization of cannabinoid CB(1) receptor in neuronal subpopulations of rat striatum: a double-label in situ hybridization study. Synapse 2000;37:71-80.

67 French ED, Dillon K, Wu X. Cannabinoids excite dopamine neurons in the ventral tegmentum and substantia nigra. Neuroreport 1997;8:649-52.

68 Gardner EL, Lowinson JH. Marijuana's interaction with brain reward systems: update 1991. Pharmacol Biochem Behav 1991;40:571-80.
69 Gessa GL, Melis M, Muntoni AL, et al. Cannabinoids activate mesolimbic dopamine neurons by an action on cannabinoid $\mathrm{CB}_{1}$ receptors. Eur J Pharmacol 1998;341:39-44.

70 Diana M, Melis M, Muntoni AL, et al. Mesolimbic dopaminergic decline after cannabinoid withdrawal. Proc Natl Acad Sci USA 1998;95:10269-73.

71 Morgan WP. Negative addiction in runners. Physician Sportsmed 1979;7:7-70.

72 Maldonado R, Rodríguez de Fonseca F. Cannabinoid addiction: behavioral models and neural correlates. J Neurosci 2002;22:3326-31.

73 Kouri EM. Does marijuana withdrawal syndrome exist? Psychiatric Times [serial online] 2002;19(2). Available from, http://www.mhsource.com/pt/ p02061.html.

74 Koob GF. Drug addiction: the yin and yang of hedonic homeostasis. Neuron 1996;16:893-6.

75 Rodríguez de Fonseca F, Carrera MR, Navarro M, et al. Activation of corticotropin-releasing factor in the limbic system during cannabinoid withdrawal. Science 2000;276:2050-4.

76 Garcia N, Jarai Z, Mirshahi F, et al. Systemic and portal hemodynamic effects of anandamide. Am J Physiol Gastrointest Liver Physiol 2001;280:G14-20.

77 Hillard CJ. Endocannabinoids and vascular function. J Pharmacol Exp Ther 2000;294:27-32

78 Wagner JA, Jarai Z, Batkai S, et al. Hemodynamic effects of cannabinoids: coronary and cerebral vasodilation mediated by cannabinoid $\mathrm{CB} 1$ receptors. Eur J Pharmacol 2001:423:203-10.

79 Zygmunt PM, Peterson J, Anderson DA, et al. Vanilloid receptors on sensory nerves mediate the vasodilator action of anandamide. Nature 1999;400:452-7.

\section{ELECTRONIC PAGES}

\section{BJSM Online case reports: http://bjsm.bmijournals.com/}

$T^{1}$ he following electronic only articles are published in conjunction with this issue of BJSM.

\section{Correlations between plasma noradrenaline concentrations, antioxidants, and neutrophil counts after submaximal resistance exercise in men \\ A Ramel, K-H Wagner, I Elmadfa}

Background: Generation of reactive oxygen species (ROS) during exercise has been linked to increased oxygen consumption. ROS could also be produced by other mechanisms-for example, a respiratory burst of neutrophils or catecholamine auto-oxidation-when oxygen consumption is only moderately increased.

Objectives: To investigate noradrenaline concentrations, neutrophil counts, plasma antioxidants, and lipid oxidation products before and after acute resistance exercise.

Methods: 17 male participants undertook a submaximal resistance exercise circuit (10 exercises; $75 \%$ of the one repetition maximum; mean (SD) exercise time, 18.6 (1.1) minutes). Blood samples were taken before and immediately after exercise and analysed for plasma antioxidants, noradrenaline, neutrophils, and lipid oxidation products. Wilcoxon's signed-rank test and Pearson's correlation coefficient were used for calculations.

Results: Neutrophils, noradrenaline, fat soluble antioxidants, and lipid oxidation products increased after exercise. Noradrenaline concentrations were associated with higher antioxidant concentrations. Neutrophils were related to higher concentrations of conjugated dienes.

Conclusions: Submaximal resistance exercise increases plasma antioxidants. This might reflect enhanced antioxidant defence in response to the oxidative stress of exercise, though this is not efficient for inhibiting lipid oxidation. The correlation between noradrenaline concentrations and plasma antioxidants suggests a modulating role of the stress hormone. Neutrophils are a possible source of oxidative stress after resistance exercise.

(Br J Sports Med 2004;38:e22) http://bjsm.bmjjournals.com/ cgi/content/full/38/5/e22

\section{Are sports medicine journals relevant and applicable to practitioners and athletes?}

C Bleakley, D MacAuley, S McDonough

Objective: To examine the evidence base of sports medicine research and assess how relevant and applicable it is to everyday practice.

Methods: Original research articles, short reports, and case reports published in four major sport and exercise medicine journals were studied and classified according to the main topic of study and type of subjects used.

Results: The most common topic was sports science, and very few studies related to the treatment of injuries and medical conditions. The majority of published articles used healthy subjects sampled from the sedentary population, and few studies have been carried out on injured participants.

Conclusions: There is a dearth of studies addressing diagnostic and treatment interventions in the sports medicine literature. The evidence base for sports medicine must continue to increase in terms of volume and quality.

(Br J Sports Med 2004;38:e23) http://bjsm.bmjjournals.com/ cgi/content/full/38/5/e23 